\title{
Síndrome de Bouveret: una rara complicación de la colelitiasis. Informe de un caso y revisión de la bibliografía
}

\author{
Bouveret's syndrome: a rarest complication of cholelithiasis. A case report and literature \\ review
}

\author{
Estephanía Navarro-del Río* y Jaime F. Hernández-Zúñiga
}

Servicio de Cirugía General, OPD Benemérito Hospital Civil de Guadalajara Fray Antonio Alcalde, Guadalajara, Jalisco, México

\begin{abstract}
Resumen
El síndrome de Bouveret se refiere a la obstrucción de la salida gástrica causada por un lito grande impactado en el duodeno que pasó a través de una fístula colecistoduodenal. Se han descrito varias técnicas endoscópicas y quirúrgicas para tratar esta entidad, pero la morbimortalidad es aún muy elevada. Se presenta el caso de una paciente femenina de 67 años con síndrome de Bouveret, con exitosa resolución mediante tratamiento quirúrgico posterior a dos tratamientos endoscópicos fallidos.
\end{abstract}

Palabras Clave: Síndrome de Bouveret. Íleo biliar. Obstrucción. Endoscopia. Cirugía.

\begin{abstract}
Bouveret's syndrome refers to the condition of gastric outlet obstruction caused by the impaction of a large gallstone into the duodenum after passage through a cholecystoduodenal fistula. Many endoscopic and surgical techniques have been described in the management of this syndrome, however the morbidity and mortality are still very high. We present the case of a 67-yearold female patient with Bouveret's syndrome, with successful resolution with surgical treatment after two failed endoscopic treatments.
\end{abstract}

Key Words: Bouveret's syndrome. Gallstone ileus. Obstruction. Endoscopy. Surgery.

\section{Correspondencia:}

*Estephanía Navarro-del Río 


\section{Introducción}

El síndrome de Bouveret es una forma infrecuente de íleo biliar, causado por el paso e impacción de un lito grande a través de una fístula colecistoduodenal; el resultado es la obstrucción de la salida gástrica'. Beaussier lo describió por primera vez en 1770. En 1896, el francés Léon Bouveret publicó los primeros dos casos de obstrucción de la salida gástrica por un lito impactado en el bulbo duodenal ${ }^{2}$.

Desde el año 2008, alrededor de 300 casos se han notificado en la bibliografía mundial. La morbilidad y mortalidad de este síndrome han descendido en los últimos años, pero aún son muy elevadas, con un cálculo de $60 \%$ y 12 a 30\%, respectivamente, según cuán oportunos sean el diagnóstico y el tratamiento".

\section{Caso clínico}

Mujer de 67 años de edad, con hipertensión arterial controlada con metoprolol y diabetes mellitus tipo II de 19 años de evolución tratada con insulina NPH (110 UI al día), en mal control, con obesidad mórbida grado II. Antecedente de poliomielitis con paraparesia en extremidades inferiores como secuela, por lo que permanece postrada. Acude a urgencias por presentar intolerancia a la vía oral y pérdida de peso. Refiere que inició su padecimiento 20 días antes con diarrea (Bristol 7), con moco sin sangre, 10 evacuaciones al día, con pérdida de peso no cuantificada, intolerancia a la vía oral con vómitos en poso de café más de cinco al día, sin dolor; acude a su hospital regional en donde se realiza gastroesofagoscopia que revela en el fondo, cuerpo y antro del estómago eritema moderado y bulbo duodenal con presencia de lesión tumoral con extensión a segunda porción del duodeno, por lo que se la envía a la institución de los autores. A su ingreso a urgencias tiene signos vitales dentro de parámetros normales y la exploración física no identifica datos relevantes. Analítica con anemia normocítica hipocrómica grado I de la OMS, glucosa de $174 \mathrm{mg} / \mathrm{dl}$, albúmina de $1.8 \mathrm{~g} / \mathrm{dl}$ y el resto dentro de cifras normales. Se solicita tomografía de abdomen con contraste intravenoso (Fig. 1) en la que se observa cámara gástrica colapsada por ausencia de contenido gástrico, una imagen de aspecto homogéneo con bordes circunscritos de aspecto calcificante hacia la izquierda de la primera y segunda porciones del duodeno que genera compresión duodenal con la cabeza del páncreas desplazada por la imagen

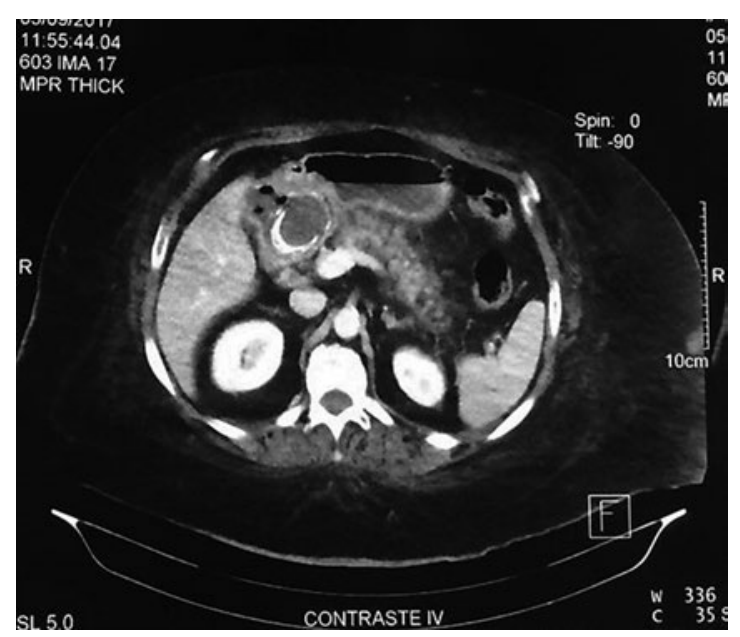

Figura 1. TC de abdomen con contraste IV que muestra vesícula biliar disminuida de tamaño, con gas en su interior y compresión por incremento del volumen del duodeno; éste muestra en su luz una imagen hipodensa con pared densa irregular parcialmente definida. TC: tomografía computarizada.

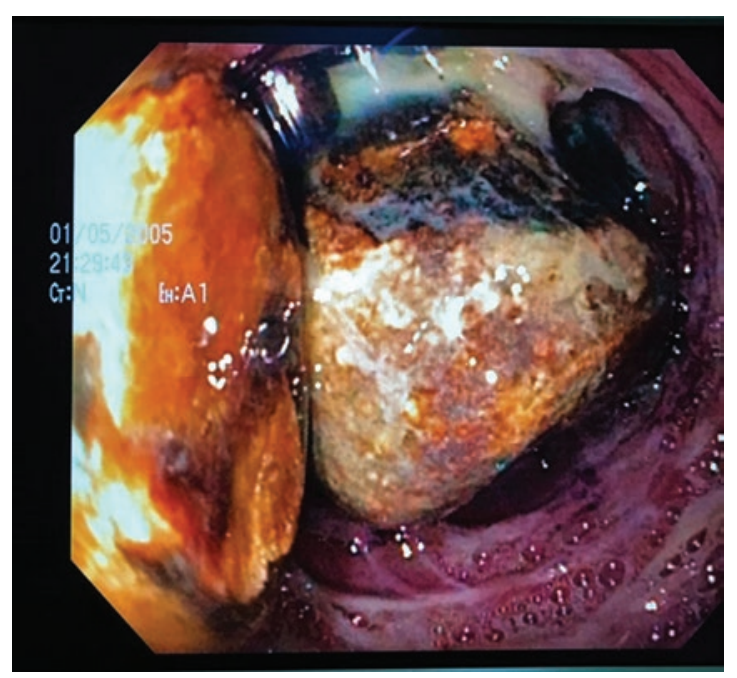

Figura 2. Gastroendoscopia. Luego del paso del píloro se observa un lito de $\sim 3.5 \mathrm{~cm}$ que obstruye el $70 \%$ de la luz e impide el paso del endoscopio.

calcificante. Se realiza gastroendoscopia (Fig. 2) en la que se reconoce después del paso del píloro un lito de $\sim 3.5 \mathrm{~cm}$ que obstruye el $70 \%$ de la luz e impide el paso del esdoscopio. Se coloca sonda nasogástrica a derivación y permanece en ayuno por intolerancia a la vía oral. Se inicia nutrición parenteral total. Durante su abordaje desarrolla infección de vías urinarias y cetoacidosis diabética, la cual se resuelve a las 48 horas. Se efectúa nueva endoscopia en la cual se logra extraer un lito de $2 \times 1 \mathrm{~cm}$ de la segunda porción del duodeno, pero permanece una concreción que no se logra extraer en la tercera porción del duodeno, por lo que se realiza laparotomía exploradora con los siguientes hallazgos: estómago adherido al 


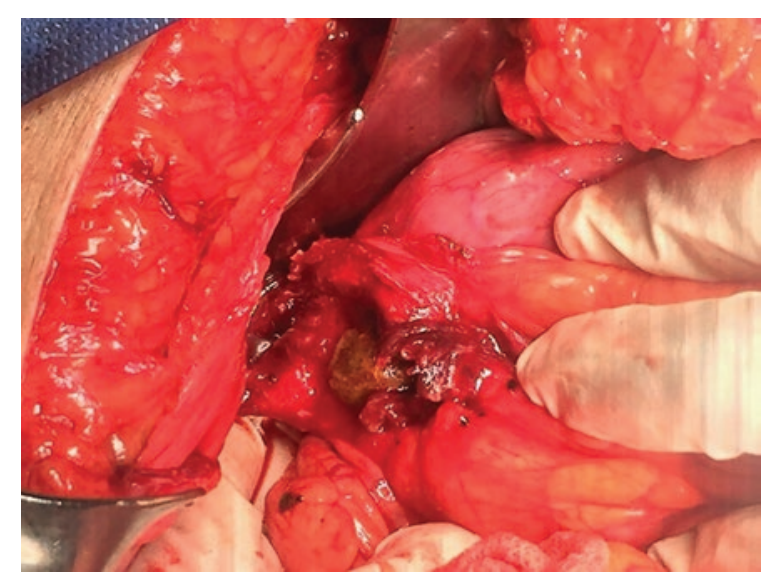

Figura 3. Fotografía transquirúrgica; se observa la fístula colecistopilórica con salida de un lito gigante.

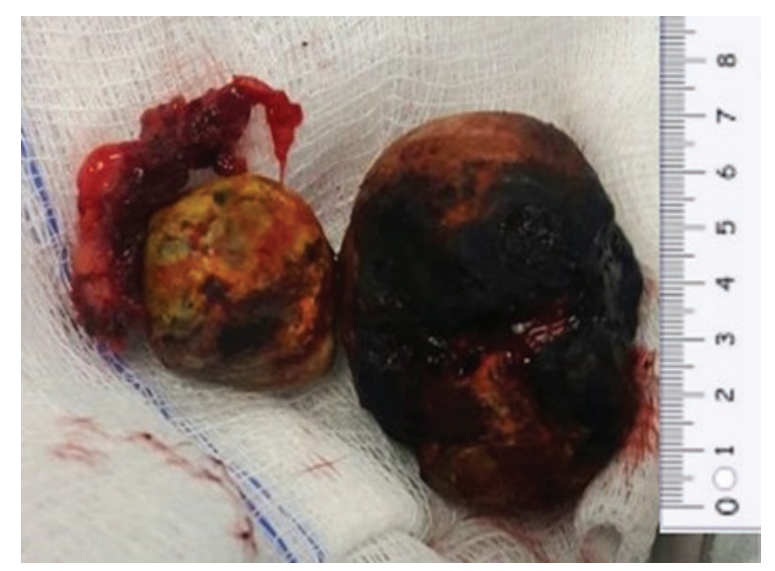

Figura 4. Litos gigantes de $3 \times 3 \mathrm{~cm}$ y $7 \times 4 \mathrm{~cm}$ extraídos de la tercera y cuarta porciones del duodeno.

hígado y vesícula biliar escleroatrófica con trayecto fistuloso hacia píloro. Se lleva a cabo una maniobra de Kocher y se palpan un cálculo de $3 \times 3 \mathrm{~cm}$ en la tercera porción del duodeno y un lito gigante de $7 \mathrm{x}$ $4 \mathrm{~cm}$ en la cuarta porción. Se abre el trayecto fistuloso y se extraen los litos (Figs. 3 y 4). Se cierra el píloro con técnica de Heineke-Mikulicz. No se logra identificar la vía biliar, por lo que se efectúa colecistectomía parcial. La paciente presenta buena evolución postquirúrgica, con inicio de la vía oral al quinto día postoperatorio. Antes del egreso se realizó gastroendoscopia en la cual no se observa trayecto fistuloso, pero se identifican úlceras duodenales en bulbo y segunda porción del duodeno (Forrest III). Recibe el alta con inhibidor de bomba de protones.

\section{Discusión}

Una fístula biliodigestiva es una rara complicación de la colelitiasis que ocurre en menos de $1 \%$ de todos los pacientes. La formación de la fístula se favorece por largos antecedentes de colelitiasis, episodios repetidos de colecistitis aguda, litos muy grandes $(2-8 \mathrm{~cm})$, sexo femenino y edad avanzada (más de 60 años). En el 60\% (53-68\%) de los casos, la fístula es colecistoduodenal' ${ }^{1}$.

El paso de los cálculos a través de la fístula puede desencadenar diferentes manifestaciones clínicas, según sean el tamaño, la parte del tracto gastrointestinal afectada y la preexistencia de zonas estenóticas a este nivel. En el $85 \%$ de todos los casos, el lito se expulsa ya sea por vómito o por las heces. Por el contrario, en el $15 \%$ de los pacientes se presenta un íleo biliar'; éste ocurre cuando una concreción de un tamaño adecuado pasa a través de una fístula colecistoduodenal o una fístula coledocoduodenal, se impacta dentro del tracto gastrointestinal, las más de las veces cerca de la unión ileocecal, y se produce una obstrucción distal del intestino delgado².

Se ha considerado que el íleo biliar aparece en el 0.3 a $0.4 \%$ de todos los pacientes con colelitiasis y es siete veces más frecuente en pacientes con edad mayor de 70 años. La localización más común, en el 50 a $90 \%$ de los casos, es el íleon terminal'. Los litos menores de $2.5 \mathrm{~cm}$ pasan casi siempre a través del intestino delgado, mientras que los más grandes se quedan impactados en la salida gástrica ${ }^{3}$.

El síndrome de Bouveret se refiere a la situación que se presenta por la impacción proximal del cálculo biliar en el duodeno o el estómago distal, lo que da lugar a la obstrucción del vaciamiento gástrico. Léon Bouveret lo describió en 1896 en dos pacientes y es extremadamente raro. Por lo general, 1 a $4 \%$ de las obstrucciones intestinales son efecto de íleo biliar y, de éstas, 1 a $3 \%$ se deben a este síndrome ${ }^{3}$.

El síndrome de Bouveret afecta con más frecuencia a mujeres de la tercera edad con una edad promedio de 68.6 años de edad ${ }^{1}$, como esta paciente, con múltiples comorbilidades. La presentación clínica puede ser inespecífica. Por lo regular, los síntomas empiezan cinco a siete días antes de acudir a la consulta médica. Se ha notificado que 43 a $68 \%$ de los pacientes tienen antecedente reciente de episodios de cólicos biliares, ictericia o colecistitis aguda, pero la primera manifestación de la colelitasis como un síndrome de Bouveret también es posible ${ }^{1}$, como en el caso de esta paciente que desconocía la presencia de colelitiasis e inició con síndrome de Bouveret.

Son frecuentes náusea, vómito y dolor abdominal en el epigastrio e hipocondrio derecho, pero la intensidad no se correlaciona casi nunca con las alteraciones anatómicas. También pueden estar presentes fiebre, 
signos de deshidratación y pérdida de peso. La sepsis es inusual. Menos habitual es que el síndrome de Bouveret se presente con hematemesis, secundaria a erosión duodenal o de la arteria celiaca.

Debido a que las manifestaciones clínicas no son tan específicas, además de la edad avanzada de los pacientes, es una situación crucial que debe considerarse, así como realizar un diagnóstico diferencial de otras causas de la obstrucción del vaciamiento gástrico, como cáncer gástrico y estenosis péptica, sobre todo en mujeres ancianas'. Esta paciente cursó con melena y fue lo que llevó a solicitar la gastroendoscopia en su primer contacto, en la cual se sospechó una tumoración neoplásica antes de establecer el diagnóstico correcto.

Para determinar el diagnóstico de síndrome de Bouveret, la triada de Rigler es patognomónica (neumobilia, litos biliares ectópicos y estómago dilatado); sin embargo, en una radiografía simple de abdomen está presente sólo en 30 a $35 \%$ de los casos ${ }^{3}$.

La mejor técnica de imagen para identificar los elementos de la triada de Rigler es la tomografía computarizada abdominal (TC). Además, la TC puede demostrar la fístula biliodigestiva por el material de contraste 0 aire, o se sospecha de manera indirecta cuando la vesícula biliar se llena de material de contraste oral; sin embargo, en el 15 a $25 \%$ de los pacientes no se logra visualizar el lito debido a la isoatenuación de la bilis y el líquido. En pacientes con emesis intensa o intolerancia al contraste oral, o en el caso de cálculos isointensos, el papel de la colangiorresonancia magnética en T2 puede ser importante ${ }^{1,2}$. También puede diagnosticarse de modo incidental por vía endoscópica².

El tratamiento óptimo para los pacientes con síndrome de Bouveret todavía es controvertido en las publicaciones mundiales. La conducta terapéutica debe de planearse tras considerar muchos parámetros, como las condiciones generales del paciente, la edad, las comorbilidades, la localización del lito, la inflamación local, el tamaño de la concreción, de la fístula y la presencia de más de un lito',4.

El objetivo primario del tratamiento es resolver la obstrucción al sustraer el lito impactado, de manera lo menos invasiva posible 1 . Debido a que es una anomalía de pacientes ancianos con comorbilidades y alto riesgo quirúrgico, el procedimiento endoscópico casi siempre es la primera línea de tratamiento.

A pesar de muchas técnicas endoscópicas disponibles, el tratamiento endoscópico sólo tiene una tasa de éxito del $9 \%{ }^{3}$. Otra situación que debe considerarse es que la fragmentación de los litos con las pinzas endoscópicas puede resultar en la migración e impacción de los fragmentos en partes distales del intestino delgado y propiciar una obstrucción intestinal de novo. Si la endoscopia falla, la operación es el tratamiento común'.

Por convención, el síndrome de Bouveret se ha tratado con laparotomía y enterotomía como el procedimiento más sencillo, con morbilidad y mortalidad relativamente elevadas, de 60 y $30 \%$ respectivamente ${ }^{1,2}$. La operación abierta, laparoscópica y laparoscópica manual se han publicado con resultados exitosos en la remoción del lito impactado. Sica, et al., informaron el primer caso de síndrome de Bouveret resuelto de modo laparoscópico ${ }^{4}$. Si es posible, el lito debe desplazarse al estómago y extraerse mediante una gastrostomía; si esto no es posible, se puede mover al intestino delgado y sustraerse a través de una enterotomía ${ }^{5}$; la intención de estas maniobras es realizar la incisión en una pared sana del órgano que, potencialmente, sana de forma adecuada. Si estas dos maniobras fallan debe efectuarse la extracción mediante una duodenotomía en la cara anterior, con especial énfasis en el cierre óptimo para evitar una estenosis'.

Aún es un tema de controversia si la colecistectomía y la reparación de la fístula deben realizarse en la misma intervención ${ }^{1}$; muchas veces es difícil y se relaciona con un alto riesgo de complicaciones y a menudo es innecesario, por lo que muchos cirujanos optan por practicar una segunda operación sólo si el paciente presenta síntomas ${ }^{2}$. Diversos estudios que comparan la combinación de colecistectomía y cierre de la fístula con la enterolitotomía sola indican que la simple extracción del lito es lo adecuado para la mayoría de los pacientes, con tasas de mortalidad de 20 a $30 \%$ y $12 \%$ respectivamente ${ }^{5}$. Además, la fístula colecistoduodenal puede funcionar como una anastomosis biliodigestiva si el tracto biliar está permeable. Otro argumento que apoya la extracción del lito por enterotomía es que los pacientes se vuelven asintomáticos después del procedimiento.

La recurrencia del íleo biliar es rara'. En este caso, la vesícula se encontraba escleroatrófica y fistulizada hacia el píloro, por lo que se decidió abrir el trayecto fistuloso para extraer los litos y realizar colecistectomía parcial (no fue posible colecistectomía total por la inflamación en la vía biliar) y reparación de la fístula con cierre del píloro.

La mortalidad postoperatoria antes del año 2000 era tan elevada como 30\%; sin embargo, el diagnóstico temprano por la endoscopia y el advenimiento de 
técnicas de mínima invasión han hecho que la mortalidad haya decrecido hasta $12 \%$.

En la institución de los autores es el primer caso de síndrome de Bouveret que se opera en al menos la última década y, pese a la mala condición general de esta paciente por sus múltiples comorbilidades, la cirugía fue exitosa, sin presentar complicaciones al año de seguimiento postoperatorio.

\section{Conflicto de intereses}

Los autores declaran que no tienen ningún conflicto de intereses.

\section{Responsabilidades éticas}

Protección de personas y animales. Los autores declaran que para esta investigación no se han realizado experimentos en seres humanos ni en animales.
Confidencialidad de los datos. Los autores declaran que han seguido los protocolos de su centro de trabajo sobre la publicación de datos de pacientes.

Derecho a la privacidad y consentimiento informado. Los autores han obtenido el consentimiento informado de los pacientes y/o sujetos referidos en el artículo. Este documento obra en poder del autor de correspondencia.

\section{Bibliografía}

1. Mavroeidis VK, Matthioudakis DI, Economou NK, Karanikas ID. Bouveret syndrome-the rarest variant of gallstone ileus: a case report and literature review. Case Rep Surg. 2013; 2013: 839370.

2. Newton RC, Loizides S, Penney N, Singh KK. Laparoscopic management of Bouveret syndrome. BMJ Case Rep. 2015; 2015. pii: bcr2015209869.

3. Baharith $\mathrm{H}$, Khan K. Bouveret syndrome: When there are no options. Can J Gastroenterol Hepatol. 2015; 29(1): 17-18.

4. Sica GS, Sileri P, Gaspari AL. Laparoscopic treatment of Bouveret's syndrome presenting as acute pancreatitis. JSLS. 2005; 9: 472-475.

5. Rodríguez-Sanjuán JC, Casado F, Fernández MJ, Morales DJ, Naranjo A. Cholecystectomy and fistula closure versus enterolithotomy alone in gallstone ileus. Br J Surg. 1997; 84: 634-637. 